\title{
NÍVEL DE DANO ECONÔMICO APLICADO À HERBOLOGIA: REVISÃO
}

\begin{abstract}
Esta revisão de literatura teve como objetivo abordar a aplicação do nível de dano econômico (NDE) no manejo de plantas daninhas. Foram discutidos os motivos para o desenvolvimento dessa relação econômica, a função do NDE como critério de decisão do controle no manejo integrado de plantas daninhas, a teoria da sua aplicação e a magnitude dos seus valores para o manejo dessas plantas, bem como as limitações e perspectivas da sua aplicação na Herbologia. O desenvolvimento de herbicidas seletivos de aplicação em pós-emergência da cultura possibilitou a adaptação do conceito do NDE para o manejo de plantas daninhas. Com isso, esse nível de dano tornou-se um dos principais fundamentos teóricos do manejo integrado, por auxiliar na tomada de decisão do controle dessas espécies nas áreas agrícolas. Na prática, o NDE é pouco utilizado no manejo de plantas daninhas por cinco motivos: (i) as relações de interferência foram desenvolvidas para organismos consumidores e produtores; (ii) ocorrência natural de populações poliespecíficas de plantas daninhas nas áreas agrícolas; (iii) falta de estabilidade das variáveis biológicas e econômicas utilizadas na sua estimativa; (iv) irrelevância do parâmetro obtido para culturas com baixa habilidade competitiva; e (v) consequências ecológicas a médio e longo prazo advindas do seu uso para o agroecossistema. Estratégias alternativas aos níveis de dano, como os programas computacionais, devem ser desenvolvidas para mediar as decisões de controle das plantas daninhas.
\end{abstract}

PALAVRAS-CHAVE: MANEJO INTEGRADO DE PLANTAS DANINHAS; NÍVEIS DE DANO; MODELOS MATEMÁTICOS.

* Engenheiro Agrônomo, Mestrando em Fitotecnia, Universidade Federal do Rio Grande do Sul (UFRGS), Porto Alegre, RS (e-mail: augustokalsing@gmail.com).

** Engenheiro Agrônomo, Ph. D. em Herbologia, Professor, Departamento de Plantas de Lavoura, UFRGS, Porto Alegre, RS, Bolsista do CNPq (e-mail: ribas.vidal@ufrgs.br). 


\section{INTRODUÇÃO}

O uso do método químico para o controle de plantas daninhas intensificou-se a partir da década de 1950, com o desenvolvimento de herbicidas com ação seletiva para as principais culturas. Desde então, o controle de plantas daninhas nas áreas agrícolas fundamentou-se na aplicação contínua e frequente desses compostos. Entretanto, alguns efeitos colaterais originaram-se do predomínio do método químico, tais como o acúmulo de resíduos nos solos e nas águas e a seleção de plantas daninhas resistentes aos herbicidas. Paralelamente, a intensificação do uso de outros pesticidas na agricultura, em especial os fungicidas e os inseticidas, também impulsionou o surgimento de efeitos adversos.

Para mitigar os problemas do método químico foram introduzidas novas abordagens para o controle de pragas. Tais abordagens foram desenvolvidas com base na integração de táticas de controle (SMITH e ALLEN, 1954), sendo inicialmente denominadas 'Controle Integrado' e, anos após, 'Manejo Integrado'. A aplicação dessa metodologia levou a adoção de novos critérios para o controle de pragas, dentre os quais se destaca o nível de dano econômico (NDE) (STERN et al., 1959). O NDE é um parâmetro que engloba fatores biológicos e econômicos, cujo valor representa a quantidade de pragas que causa dano equivalente ao custo do seu controle. Na prática, esse critério técnico consiste na análise de custo:benefício que permite otimizar o retorno econômico de determina tática de controle de pragas.

O desenvolvimento de herbicidas seletivos de aplicação em pós-emergência, no final da década de 1970, oportunizou a adaptação do NDE para o manejo de plantas daninhas (COUSENS, 1985; 1987). Desde então, fomentou-se a sua aplicação nos programas de Manejo Integrado de Plantas Daninhas (MIPD), sobretudo para as culturas de arroz, batata, milho, trigo e soja. Contudo, os agricultores e extensionistas têm sido pouco propensos a utilizá-lo como critério para auxiliar a decisão sobre o manejo das infestantes (HAMMOND et al., 2006; WILSON et al., 2009). As causas pelas quais o conceito do NDE apresenta-se subutilizado para o manejo de infestantes são ainda pouco compreendidas.

Os objetivos desta revisão de literatura foram: apresentar e discutir os fundamentos do NDE; avaliar e discutir as limitações da aplicabilidade do NDE para o manejo das plantas daninhas; e, apresentar as novas abordagens e perspectivas do uso do NDE para o manejo das infestantes.

\section{MANEJO INTEGRADO DE PLANTAS DANINHAS: A TEORIA}

O agroecossistema consiste no local com produção agrícola em que a ação antrópica altera o ecossistema (GLIESSMAN, 2001). Nesses locais estão inseridas as plantas cultivadas, as plantas daninhas e os demais componentes bióticos, entre os quais ocorrem relações de interferência (VIDAL, 2010). Desde a antiguidade, os humanos intervêm nesse ambiente com o intuito de reduzir parcial ou totalmente a interferência das plantas daninhas sobre as plantas de seu interesse. Assim, criaram formas de manejá-las, utilizando desde o arranquio manual, enxadas, até diversos métodos de controle culturais, físicos e químicos (TIMMONS, 2005). Essas práticas são utilizadas tanto de forma associada como isolada, de acordo com a estratégia de intervenção utilizada para gerir a comunidade daninha.

Durante duas décadas, a partir de 1960, o controle de plantas daninhas teve como objetivo único a erradicação permanente dessas espécies das áreas agrícolas. Erradicar, naquela época, tinha o sentido de eliminar completamente quaisquer espécies de infestantes (COUSENS, 1987). Para isso, as estratégias de controle de plantas daninhas fundamentavam-se na utilização contínua de herbicidas, geralmente com produtos de ação residual (HAMILL, 2004). Contudo, verificou-se com o passar do tempo que a estratégia de erradicação química não tinha capacidade para eliminar inteiramente as plantas daninhas das áreas cultivadas. Tal constatação sugeria o desenvolvimento 
de nova perspectiva de intervenção antrópica no agroecossistema para manejar a comunidade infestante.

A partir da década de 1970, o desenvolvimento de herbicidas de aplicação em pósemergência possibilitou a criação das estratégias de contensão e redução da densidade de plantas daninhas (BUHLER, 2002). Nessas categorias de intervenção considera-se aceitável algum nível de perda de produtividade e, em vez de se almejar o controle absoluto das infestantes, passou-se a dar importância à manutenção da sua densidade populacional abaixo do NDE. Nesse caso, as plantas daninhas são monitoradas na estação de crescimento e as medidas de redução da sua população são utilizadas quando necessário (FLECK et al., 2002b). Assim, o NDE constitui um dos principais fundamentos do MIPD por auxiliar os produtores na decisão do controle dessas plantas nas áreas agrícolas (Figura 1).

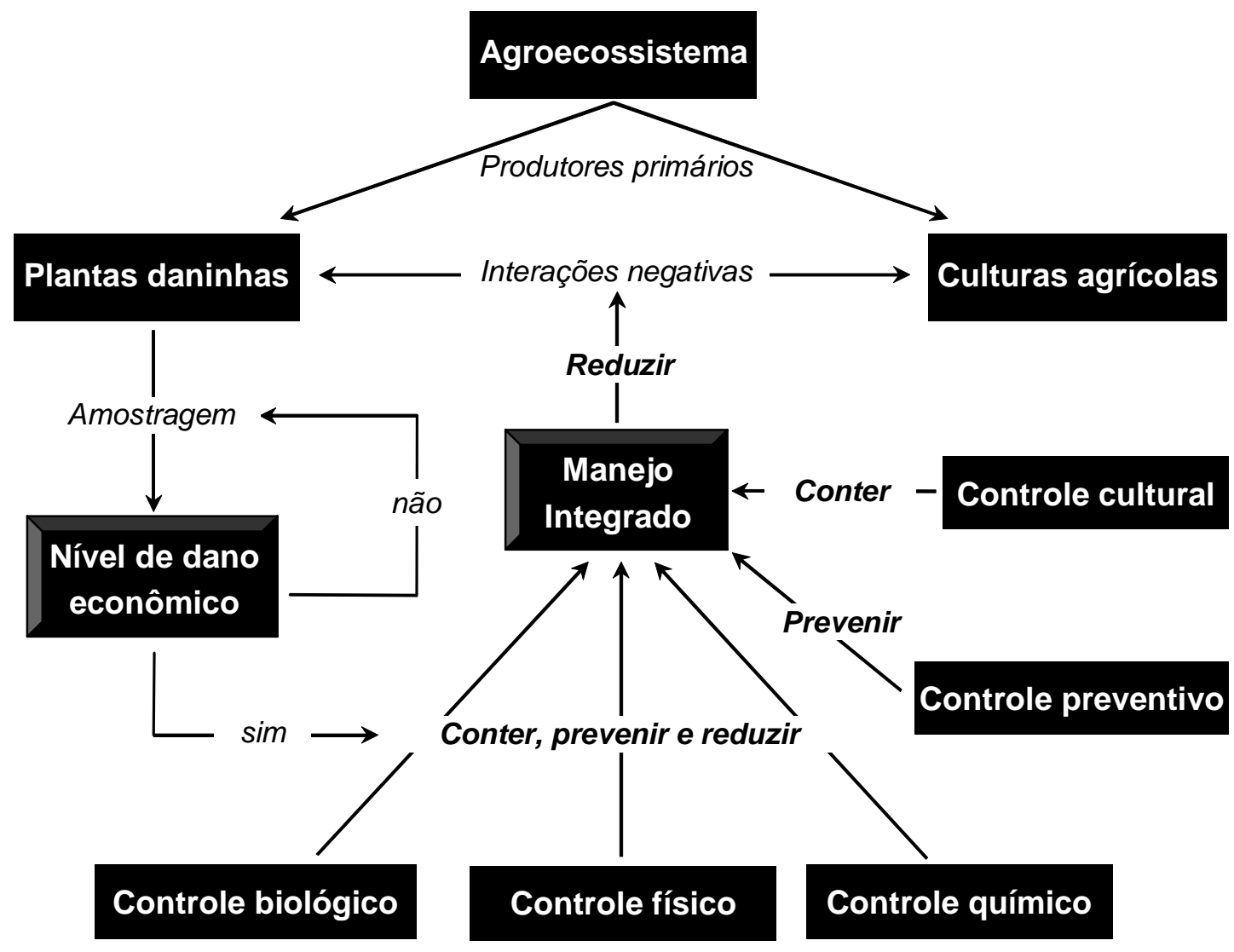

\section{FIGURA 1 - MAPA CONCEITUAL PARA A ESTRUTURA DO MANEJO INTEGRADO DE PLANTAS DANINHAS NO AGROECOSSISTEMA}

O MIPD pode ser definido como o conjunto de estratégias de contensão, prevenção e redução da população de plantas daninhas, mantendo-a abaixo de determinado NDE (SWANTON e WEISE, 1991; BUHLER, 2002). Na sua abordagem também se consideram análises de custo: benefício que avaliam o interesse e o impacto das estratégias de controle no ambiente e na sociedade. Somente o uso de múltiplas estratégias de controle não podem caracterizar o MIPD, mas sim a relação dessas, tendo como base fundamentos ambientais, agronômicos, 
econômicos e sociais (SWANTON e MURPHY, 1996; SANYAL et al., 2008). Pode-se considerar a proposta do MIPD, portanto, como a mais ampla e mais complexa até então desenvolvida para a gestão das plantas daninhas.

Na prática, o MIPD consiste em diversas ações, dentre as quais algumas são consideradas de suma importância: (i) criar ambientes adversos para as plantas daninhas por meio da alteração do ambiente; (ii) reduzir a produção de sementes (ou propágulos) dessas espécies na área cultivada; e (iii) intervir na densidade populacional de modo a mantê-la abaixo de determinado NDE (Figura 1). Ressalta-se que a execução dessas ações requer o conhecimento de pelo menos três fundamentos básicos. O primeiro envolve as características ecológicas das espécies daninhas ocorrentes na área cultivada; o segundo as relações de interferência entre essas espécies e as culturas agrícolas; e o terceiro os custos e benefícios das distintas estratégias que possam ser empregadas para o manejo das infestantes.

\section{APLICAÇÃO DO NÍVEL DE DANO ECONÔMICO NA HERBOLOGIA}

Nível de dano pode ser definido como o limite a partir do qual se gera uma resposta. Na agricultura, esse termo tem sido considerado sob distintas perspectivas, especialmente, para designar a densidade populacional de um agente nocivo que causa dano a uma cultura. O NDE apresenta-se, dentre os demais níveis de dano, como aquele de maior ênfase na agricultura. A definição mais aceita para o NDE foi apresentada como "a mais baixa densidade populacional de uma praga que resulta em dano econômico" (STERN et al., 1959). Destaca-se que esse conceito foi inicialmente desenvolvido para o manejo de insetos, sendo posteriormente adaptado para programas de manejo de plantas daninhas.

Sob o ponto de vista do MIPD, a interferência de qualquer espécie daninha na produção de determinada cultura provoca dano, que pode ser definido como a redução do seu rendimento. Esse dano pode ser quantificado monetariamente como a perda de receita da cultura, a qual recebe o nome de dano econômico. A realização desse cálculo envolve o conhecimento de alguns parâmetros agronômicos e econômicos, conforme descrito na Equação 1:

$$
\mathrm{DE}=(\mathrm{NCD}) \star(\mathrm{D}) \star(P)^{\star}(\mathrm{Q}) \quad \text { (Equação 1) }
$$

Em que:

$\mathrm{DE}=$ dano econômico à cultura $\left(\mathrm{R} \$ \mathrm{ha}^{-1}\right) ; \mathrm{NCD}=$ nível crítico de dano $(\%) ; \mathrm{N}=$ densidade da planta daninha (plantas $\left.\mathrm{m}^{-2}\right) ; \mathrm{P}=$ preço do produto $\left(\mathrm{R} \$ \mathrm{~kg}^{-1}\right)$; e $\mathrm{Q}=$ produção da cultura na ausência de controle $\left(\mathrm{Kg} \mathrm{ha}^{-1}\right)$ (VIDAL et al., 2010a).

A atividade agrícola, como qualquer empreendimento, deve remunerar o agricultor pelo seu investimento e trabalho. Assim, a aplicação de medida de controle das plantas daninhas envolve alguns custos que devem ser minimizados e a receita bruta que deve ser maximizada. O lucro bruto de determinada medida de controle pode ser estimado, conforme descrito na Equação 2:

$$
\mathrm{L}=\left[(\mathrm{NCD})^{\star}(\mathrm{D})^{\star}(\mathrm{P})^{\star}(\mathrm{Q})\right]-(\mathrm{C}) \quad \text { (Equação 2) }
$$

Em que:

$\mathrm{L}=$ lucro bruto $\left(\mathrm{R} \$ \mathrm{ha}^{-1}\right)$; e $\mathrm{C}$ = custo de controle das plantas daninhas, incluindo-se a aplicação dessa medida de controle.

O lucro bruto é maximizado quando as curvas da receita bruta e do custo da medida de controle das plantas daninhas afastarem-se o máximo, ou seja, quando a derivada do lucro bruto em 
função de determinada medida de controle das infestantes $(x)$ for igual a zero, conforme descrito na Equação 3:

$$
[\mathrm{dL} / \mathrm{d}(\mathrm{x})]=\mathrm{P}[\mathrm{dQ} / \mathrm{d}(\mathrm{x})]-[\mathrm{dC} / \mathrm{d}(\mathrm{x})]=0 \quad \text { (Equação 3) }
$$

Obtém-se então o NDE, ou seja, a densidade de plantas daninhas que proporciona o máximo retorno econômico de determinada medida de controle. Na prática, essa relação econômica indica quando o impacto causado pela densidade de plantas daninhas apresenta valor equivalente ao custo do seu controle. Assim, esse critério técnico representa uma relação de custo: benefício da aplicação de determinada medida de controle, conforme descrito na Equação 4:

$$
\mathrm{NDE}=(\mathrm{C}) /\left[(\mathrm{NCD})^{\star}(\mathrm{D})^{\star}(\mathrm{P}) *(\mathrm{Q})^{\star}(\mathrm{E})\right] \quad \text { (Equação 4) }
$$

Em que:

$\mathrm{NDE}=$ nível de dano econômico da espécie daninha $\left(\right.$ plantas $\left.\mathrm{m}^{-2}\right) ; \mathrm{e}, \mathrm{E}=$ eficácia da medida adotada para o controle das plantas daninhas (\%).

Destaca-se que o NDE assim definido apresenta alguns pressupostos básicos. Primeiro, assume-se que os parâmetros NCD e E sejam previsíveis, ou seja, que a seu valor estimado represente o cenário real. No segundo pressuposto admite-se que a cultura e a planta daninha consideradas para o cálculo sofrem poucos efeitos da interferência exercida por outras espécies de plantas daninhas. Finalmente, os custos e benefícios da medida de controle dizem respeito apenas ao ano considerado.

\section{MAGNITUDE DO NÍVEL DE DANO ECONÔMICO DE PLANTAS DANINHAS}

Ao longo das últimas duas décadas têm-se ampliado os estudos de avaliação de NDE para plantas daninhas (PORTUGAL e VIDAL, 2009). Em pesquisa realizada na base de dados da plataforma de periódicos Web of Science (ISI, 2010), com os termos 'planta daninha' (weed) e 'nível de dano econômico' (economic threshold) verificou-se que mais de uma centena de artigos científicos abordam esse assunto. $A$ análise dos estudos publicados nos principais periódicos da área de plantas daninhas permite considerar a magnitude dos NDE de plantas daninhas obtidos em culturas de lavoura e hortícolas (Tabelas 1, 2 e 3).

A maioria dos estudos tem avaliado NDE para culturas de estação estival, sobretudo para cultivos de arroz, girassol, milho e soja. Os valores médios desse critério encontram-se entre quatro a seis plantas $\mathrm{m}^{-2}$ para o girassol, menos de cinco plantas $\mathrm{m}^{-2}$ para milho e menos de uma planta $\mathrm{m}^{-2}$ para arroz e soja. Valores similares foram obtidos para as culturas de algodão, ervilha e feijão, nos quais os NDE foram inferiores a dez plantas $\mathrm{m}^{-2}$ na maior parte das situações (Tabela 1).

Os valores de NDE para culturas de estação hibernal, cevada e trigo, indicam que as mesmas apresentam elevada habilidade competitiva com as infestantes. Assim, os NDE nessas culturas têm geralmente valores superiores aqueles obtidos para os cereais de estação estival. Os valores médios encontram-se entre 25 a 40 plantas $^{-2}$ para ambas as culturas, o que demonstra o potencial desse critério técnico para o manejo de plantas daninhas nesses cereais (Tabela 2).

Os valores de NDE obtidos para culturas hortícolas, em especial nos cultivos de batata, cebola, cenoura e tomate indicam que as mesmas apresentam reduzida habilidade competitiva com as plantas daninhas. Os valores médios de NDE nessas culturas são geralmente inferiores a uma planta $\mathrm{m}^{-2}, \mathrm{o}$ que demonstra o elevado impacto negativo da interferência das infestantes em culturas hortícolas (Tabela 3). Destaca-se que para essas culturas há muito menos estudos sobre NDE em relação às demais, o que representa grande oportunidade de pesquisa em aberto. 


\section{TABELA 1 - NÍVEL CRÍTICO DE DANO (NCD) E NÍVEL DE DANO ECONÔMICO (NDE) DE PLANTAS DANINHAS PARA CULTURAS DE LAVOURA DE ESTAÇÃO ESTIVAL}

\begin{tabular}{|c|c|c|c|c|c|}
\hline Cultura & Espécie daninha & $\mathrm{NCD}^{1}$ & $\mathrm{NDE}^{2}$ & Referência $^{3}$ & Ano \\
\hline Algodão & Zea mays & 5,0 a 9,0 & 0,02 a 0,08 & THOMAS et al. & 2007 \\
\hline Algodão & Zea mays & 4,0 a 8,0 & 0,02 a 0,07 & CLEWIS et al. & 2008 \\
\hline Arroz & Echinochloa spp. & 0,3 a 24,6 & 0,1 a 13,4 & LINDQUIST et al. & 1996 \\
\hline Arroz & Echinochloa spp. & 4,7 a 29,3 & 0,6 a 3,1 & GALON et al. & 2007 \\
\hline Arroz & Oryza sativa & 0,3 a 12,7 & 0,3 a 38,0 & AGOSTINETTO et al. & 2005 \\
\hline Arroz & Sagitaria montevidensis & 0,0 a 0,4 & 0,0 a 200,0 & GIBSON et al. & 2001 \\
\hline Beterraba & Echinochloa-crus-galli & 1,9 a 12,7 & 0,05 a 0,2 & NORRIS, R. F. & 1992 \\
\hline Ervilha & Avena sativa & 0,3 a 7,2 & 0,7 a 14,9 & LUTMAN et al. & 1994 \\
\hline Ervilha & Cevada silvestre & 1,7 a 5,4 & 2,0 a 6,0 & ODONOVAN \& BLACKSHAW & 1997 \\
\hline Feijão & Avena sativa & 0,3 a 7,2 & 3,9 a 12,7 & LUTMAN et al. & 1994 \\
\hline Feijão & Digitaria sanguinalis & 9,3 a 38,7 & 0,0 a 1,0 & AGUYOH e MASIUNAS & 2003 \\
\hline Girassol & Ammi majus & 1,1 a 1,8 & 4,0 a 6,0 & ONOFRI et al. & 1994 \\
\hline Girassol & Chenopodium album & 1,1 a 1,8 & 4,0 a 6,0 & ONOFRI et al. & 1994 \\
\hline Girassol & Ridolfia segetum & 0,0 a 0,1 & 1,0 a 2,5 & CARRANZA et al. & 1995 \\
\hline Girassol & Sinapis arvensis & 1,1 a 1,8 & 4,0 a 6,1 & ONOFRI et al. & 1994 \\
\hline Girassol & Orobanche cernua & 0,2 a 0,4 & 1,5 a 3,5 & GARCIATORRES et al. & 1996 \\
\hline Milho & Abutilon theophrasti & 3,7 & 0,3 a 1,7 & SATTIN et al. & 1992 \\
\hline Milho & Abutilon theophrasti & 1,8 a 60,3 & 0,1 a 14,0 & CARDINA et al. & 1995 \\
\hline Milho & Abutilon theophrasti & 3,4 & 1,0 a 8,5 & WERNER et al. & 2004 \\
\hline Milho & Amaranthus retroflexus & 3,0 a 16,5 & 0,6 a 5,3 & KNEZEVIC et al. & 1994 \\
\hline Milho & Brachiaria plantaginea & 0,0 a 0,03 & 0,2 a 2,0 & VIDAL et al. & 2004 \\
\hline Milho & Chenopodium album & 0,7 a 10,5 & 0,3 a 4,2 & FISCHER et al. & 2004 \\
\hline Milho & Echinochloa crus galli & 0,3 & 6,4 & BOSNIC e SWANTON & 1997 \\
\hline Milho & Setaria spp. & 0,1 a 2,5 & 3,1 a 94,1 & LINDQUIST et al. & 1999 \\
\hline Milho & Setaria viridis & 0,5 a 10,0 & 6,0 a 167,0 & CATHCART \& SWANTON & 2003 \\
\hline Soja & Abutilon theophrasti & 2,1 a 4,2 & 0,2 a 0,4 & BAUER et al. & 1991 \\
\hline Soja & Abutilon theophrasti & 0,03 & 0,3 & BAUER e MORTENSEN & 1992 \\
\hline Soja & Amaranthus tuberculatus & 6,5 & 0,2 & BAUER et al. & 1991 \\
\hline Soja & Ambrosia artemisiifolia & 9,9 a 28,6 & 0,0 a 0,1 & COWBROUGH et al. & 2003 \\
\hline Soja & Ambrosia trifida & 5,2 a 7,3 & 0,3 & BAYSINGER e SIMS & 1991 \\
\hline Soja & Bidens spp. & 0,4 a 13,5 & 0,4 a 33,0 & RIZZARDI et al. & 2003 \\
\hline Soja & Helianthus annuus & 1,4 & 0,6 & BAUER et al. & 1991 \\
\hline Soja & Helianthus annuus & 0,05 & 0,2 & BAUER e MORTENSEN & 1992 \\
\hline Soja & Sida rhombifolia & 0,2 a 2,2 & 2,0 a 50,0 & FLECK et al. & $2002 b$ \\
\hline
\end{tabular}

\footnotetext{
1. Valores percentuais (perda planta-1 $\mathrm{m}^{-2}$ ).

2-Valores em unidades de indivíduos (plantas $\mathrm{m}^{-2}$ ).

3- Valores obtidos nos periódicos Planta Daninha, Weed Research, Weed Science e Weed Technology, em pesquisa realizada na plataforma Web of Science com trabalhos publicados entre 1990 e 2010.
} 


\section{TABELA 2 - NÍVEL CRÍTICO DE DANO (NCD) E NÍVEL DE DANO ECONÔMICO (NDE) DE PLANTAS DANINHAS PARA CULTURAS DE LAVOURA DE ESTAÇÃO HIBERNAL}

\begin{tabular}{|c|c|c|c|c|c|}
\hline Cultura & Espécie daninha & $\mathrm{NCD}^{1}$ & $\mathrm{NDE}^{2}$ & Referência $^{3}$ & Ano \\
\hline Canola & Avena sativa & 0,0 a 2,3 & 2,3 a 72,5 & LUTMAN et al. & 1994 \\
\hline Cevada & Avena sativa & 0,0 a 2,3 & 10,9 a 18,4 & LUTMAN et al. & 1994 \\
\hline Trigo & Alopecurus myosuroides & -4 & 25,0 a 35,0 & ZANIN et al. & 1993 \\
\hline Trigo & Avena sterilis & - & 7,0 a 12,0 & ZANIN et al. & 1993 \\
\hline Trigo & Bromus sterilis & - & 0,0 a 2,0 & ZANIN et al. & 1993 \\
\hline Trigo & Lolium multiflorum & - & 25,0 a 35,0 & ZANIN et al. & 1993 \\
\hline Trigo & Secale cereale & 0,4 a 19,9 & 0,9 a 11,8 & PESTER et al. & 2000 \\
\hline Trigo & Vicia sativa & - & 5,0 a 10,0 & ZANIN et al. & 1993 \\
\hline
\end{tabular}

\section{TABELA 3 - NÍVEL CRÍTICO DE DANO (NCD) E NÍVEL DE DANO ECONÔMICO (NDE) DE PLANTAS DANINHAS PARA CULTURAS HORTÍCOLAS}

\begin{tabular}{llccll}
\hline Cultura & Espécie daninha & NCD $^{1}$ & NDE $^{2}$ & \multicolumn{1}{c}{ Referência $^{3}$} & Ano \\
\hline Batata & Elytrigia repens & 0,08 a 0,3 & 0,04 a 2,0 & BAZIRAMAKENGA e LEROUX & 1998 \\
Brócolis & Lolium perenne & 0,5 a 2,2 & 1,7 a 7,3 & BELL, C. E. & 1995 \\
Cebola & Solanum tuberosum & 16,5 & 0,03 & WILLIAMS et al. & 2004 \\
Cenoura & Solanum tuberosum & 87,0 & 0,03 a 0,13 & WILLIAMS e BOYDSTON & 2006 \\
Tomate & Echinochloa spp. & 1,3 a 13,3 & 0,04 a 0,06 & NORRIS et al. & 2001 \\
Tomate & Solanum nigrum & 3,0 a 23,0 & 0,5 a 2,0 & PORTUGAL, J. & 2010 \\
Tomate & Solanum ptycanthum & 11,6 & 0,04 & BUCKELEW e MONKS & 2006 \\
\hline
\end{tabular}

1- Valores percentuais (perda planta-1 $\mathrm{m}^{-2}$ ).

2-Valores em unidades de indivíduos (plantas $\mathrm{m}^{-2}$ ).

3-Valores obtidos nos periódicos Planta Daninha, Weed Research, Weed Science e Weed Technology, em pesquisa realizada na plataforma Web of Science com trabalhos publicados entre 1990 e 2010.

\section{LIMITAÇÕES DA APLICAÇÃO DO NÍVEL DE DANO ECONÔMICO PARA O MANEJO DE PLANTAS DANINHAS}

Ações de extensão, iniciadas a partir da década de 1980, têm fomentado a introdução dos NDE para o manejo de plantas daninhas. Contudo, esse conceito tem sido pouco utilizado como critério para o manejo dessas espécies. Especula-se que a subutilização do NDE para o manejo de plantas daninhas ocorra por pelo menos cinco motivos: (i) relações de interferência entre organismos consumidores e produtores no agroecossistema; (ii) ocorrência natural de populações poliespecíficas de plantas daninhas nas áreas agrícolas; (iii) estabilidade das variáveis biológicas e das econômicas empregadas para a sua estimativa; (iv) irrelevância do parâmetro obtido para culturas com baixa habilidade competitiva com infestantes; e, (v) implicações ecológicas a médio e longo prazo advindas do seu uso nas áreas cultivadas. A seguir serão detalhados os principais aspectos dessas limitações. 


\subsection{RELAÇÕES ENTRE ORGANISMOS}

O NDE foi inicialmente desenvolvido para modelar a relação de interferência entre determinada espécie de inseto e uma cultura. Nessa condição, há clara relação de interferência entre um organismo consumidor (inseto) e um produtor (cultura), ou seja, existe relação de causa e efeito entre a densidade de insetos e o dano à cultura. Como as plantas daninhas também são organismos produtores, não há relação direta de causa e efeito entre a densidade dessas plantas e o rendimento agronômico da cultura. Além disso, podem ocorrer interações com outros organismos (insetos, patógenos, nematóides), fato que dificulta ainda mais a estimativa da interação entre cada planta daninha com relação ao rendimento agronômico da cultura avaliada.

O aspecto conceitual constitui o principal limitador da utilização do NDE para o manejo de plantas daninhas (VIDAL, 2010a). A baixa habilidade em prever o dano agronômico causado pela população de plantas daninhas compromete fortemente a aplicação desse critério de decisão na Herbologia. Quando muito, têm-se obtido relações semi-empíricas que relacionem ambas as variáveis, ou seja, relações que apresentem significado biológico nos seus parâmetros.

\subsection{POPULAÇÕES POLIESPECÍFICAS}

O NDE de plantas daninhas consiste de um modelo com caráter monoespecífico (NORRIS, 1999), ou seja, foi desenvolvido para modelar as relações entre uma espécie de planta daninha e uma cultura. A aplicação desse critério será limitada, portanto, a apenas uma espécie infestante. Todavia, nas condições reais do agroecossistema, as infestações são constituídas por várias espécies vegetais. Assim, os estudos monoespecíficos geralmente não refletem as situações usuais de áreas cultivadas em que ocorrem interações das infestantes entre si e dessas com as plantas cultivadas (VIDAL et al., 2010b).

Os métodos de cálculo do NDE para populações poliespecíficas são distintos daquele apresentado nesta revisão. Tais métodos estão descritos em detalhes em recente revisão de PORTUGAL e VIDAL (2009) e não serão reapresentados aqui. Cabe destacar que a determinação do NDE para populações poliespecíficas também apresenta algumas limitações, em especial, a estimativa da interação interespecífica entre espécies de plantas daninhas (SWANTON e WEISE, 1991). Outra dificuldade, refere-se ao reduzido número de estudos realizados para a determinação do NDE tendo como base duas ou mais espécies de plantas daninhas (VIDAL et al., 2010b).

\subsection{ESTABILIDADE DAS VARIÁVEIS BIOLÓGICAS}

Os valores das variáveis biológicas usadas para a determinação do NDE não apresentam estabilidade entre os anos agrícolas e locais da mesma região. De fato, uma das principais dificuldades em se usar o NDE é que o produtor não conhece antecipadamente qual será o rendimento da cultura na ausência dessas espécies (FLECK et al., 2002b). Na prática, o valor dessa variável é apenas estimado, tendo como base o histórico de produtividade da área cultivada ou a meta de rendimento para a qual a cultura é manejada. Outra limitação do NDE envolve a variação do valor do nível crítico de dano (NCD) das plantas daninhas no tempo e no espaço. Diversos trabalhos demonstram que o valor desse índice apresenta ampla variabilidade no agroecossistema (BAUER et al., 1991; LINDQUIST et al., 1996, 1999; McDONALD e RIHA, 1999; PESTER et al., 2000; FISCHER et al., 2004; VIDAL et al., 2010a). Mesmo modelos mecanísticos, que envolvem fundamentos e princípios da fisiologia da produção agrícola vegetal, não são suficientemente precisos para estimar com exatidão a perda de produção da cultura proporcional por planta daninha.

A interação entre diversos fatores torna complexa a determinação do impacto das infestantes no rendimento da cultura. Dentre esses fatores destacam-se o espaçamento da cultura (AGOSTINETTO et al., 2005), a época de emergência da cultura em relação às plantas daninhas (RIZZARDI et al., 
2003) e aspectos do agroecossistema como o sistema de preparo do solo (CARDINA et al., 1995) e o seu nível de fertilidade (CATHCART e SWANTON, 2003), entre outros. Esses estudos demonstram que o ambiente e as práticas de manejo da cultura têm elevada influência nas relações de interferência interespecíficas, as quais podem assumir importância superior à da densidade de infestantes na redução do rendimento da cultura.

\subsection{ESTABILIDADE DAS VARIÁVEIS ECONÔMICAS}

As variáveis econômicas do NDE (custo do controle e preço do produto) diferem com o tempo em função das oscilações econômicas do mercado. De fato, as matérias-primas industriais (insumos) e as commodities agrícolas (produtos agrícolas) apresentam elevada volatilidade no mercado, sobretudo, pela variação nas relações de oferta e demanda. Assim, no início da safra, essas variáveis podem apresentar nível de preço diferente daquele revelado na época da colheita, o que levaria à tomada de decisão do controle das plantas daninhas com estimativa de lucro incorreta pelos agricultores (SWANTON et al., 1999).

Da mesma forma, o NDE calculado em determinada safra raramente pode ser utilizado em tomadas de decisão para o controle nas safras seguintes, pois esse nível varia conforme as alterações ocorridas na economia (VIDAL et al., 2004). A incerteza sobre o preço dos produtos agrícolas e dos custos das medidas de controle tende a diminuir os valores do NDE, fato que conflita com a baixa tolerância ao risco econômico dos agricultores (SWINTON, 1991).

\subsection{IRRELEVÂNCIA DO PARÂMETRO COMO CRITÉRIO TÉCNICO}

Os NDE de plantas daninhas para culturas com baixa habilidade competitiva são menores do que as populações usuais dessas espécies que ocorrem nas áreas cultivadas. Com efeito, os valores de NDE para a maioria das culturas são geralmente inferiores a uma planta $\mathrm{m}^{-2}$, o que limita a sua aplicação como critério de controle de infestantes. No caso de culturas hortícolas, por exemplo, os valores desse parâmetro são centesimais, o que torna desnecessária a sua utilização na maioria dos casos.

As infestações de plantas daninhas ocorrem de forma elevada, pandêmica e crônica (VIDAL et al., 2010a). Assim, poucos dias após a emergência da cultura, a lavoura apresenta densidade de infestação muito superior ao valor estimado de NDE, o que torna esse critério irrelevante na orientação do controle. Esse fato constitui uma das maiores justificativas dos produtores para desuso do NDE, devido o seu conhecimento empírico da elevada infestação das lavouras com as infestantes.

\subsection{CONSEQUÊNCIAS ECOLÓGICAS A MÉDIO E LONGO PRAZO}

As plantas daninhas são organismos endêmicos e, como tal, regeneram-se a partir de sementes do solo (NORRIS, 1999). Deste modo, as plantas remanescentes nas áreas cultivadas tendem a abastecer o banco de sementes e a aumentar ainda mais o problema com plantas daninhas no sistema de cultivo. Essas implicações podem, inclusive, afetar as relações de arrendamento das propriedades agrícolas por ocasião da utilização do conceito do NDE para o manejo de infestantes. De fato, alguns produtores expressam cláusulas nos contratos de arrendamento que preveem multas aos locatários em decorrência da infestação da área com plantas daninhas.

Cabe destacar que alguns autores apóiam a redução do NDE para outro nível, denominado nível de dano econômico ótimo (NDEO), que considera a produção de sementes das plantas daninhas (BAUER e MORTENSEN, 1992; FLECK et al., 2002b). Contudo, a aplicação do NDEO na Herbologia mitigaria apenas as consequências ecológicas a médio e longo prazo, sendo as demais limitações apresentadas anteriormente comuns a ambos os níveis de dano na área de plantas daninhas. 


\section{PERSPECTIVAS DA APLICAÇÃO DO NÍVEL DE DANO ECONÔMICO PARA O MANEJO DE PLANTAS DANINHAS}

As diversas limitações apresentadas no tópico anterior têm minimizado a adoção do NDE como critério que oriente a decisão do controle das plantas daninhas. Para contornar essa situação, duas propostas clássicas buscam aumentar a aplicabilidade desse critério na Herbologia. Essas propostas incluem o aumento da capacidade de previsão do nível crítico de dano (NCD) e a avaliação das consequências ecológicas da utilização dos níveis de dano. Cabe destacar que as discussões e perspectivas já relatadas para essa última proposta, tais como em NORRIS (1992), BAUER e MORTENSEN (1992) e FLECK et al.( 2002a), entre outros, também são pertinentes ao assunto desse tópico e não serão revistas aqui em decorrência da sua extensa descrição na literatura da área de plantas daninhas.

A quantificação da perda proporcional por unidade de planta daninha, ou NCD, apresenta difícil estimativa devido à variabilidade associada com o agroecossistema e efeitos dos sistemas de cultivo, como mencionado. As abordagens de aumento da previsibilidade dessa variável seguem duas linhas: (i) o uso de diferentes variáveis explicativas da interferência entre infestantes e culturas, e (ii) o uso de duas ou mais dessas variáveis explicativas por meio de modelos lineares múltiplos.

A primeira abordagem baseia-se no pressuposto que a densidade de plantas não apresenta relação satisfatória com o rendimento da cultura, ou seja, de que essa relação apresenta baixo significado biológico. De fato, nos estudos de avaliação do NCD não é considerado que as populações de plantas daninhas são compostas por uma hierarquia entre os indivíduos (LUTMAN et al., 1996; 2000), ou tampouco que existem diferenças morfológicas entre os mesmos. Portanto, os modelos semi empíricos usados para relacionar a interação entre plantas daninhas e cultivadas com base na densidade subestimam o impacto dessas espécies infestantes nas culturas. Existe, no entanto, a perspectiva de uso de outras variáveis, que se baseiam nas características das plantas ou na estrutura do dossel, as quais representam com maior precisão as características de uma população. Essas variáveis incluem a cobertura vegetal do solo, as massas fresca e desidratada da parte aérea e das raízes das plantas e o seu índice de área foliar, entre outras. Modelos contendo essas variáveis têm atingido maior precisão na quantificação da interferência em relação àqueles baseados na densidade de plantas (FLECK et al., 2002a) e poderiam ser usados no aumento da previsão nos modelos de perda da produção agrícola.

A segunda abordagem baseia-se no fato de que quanto maior for o número de variáveis explicativas para determinado fenômeno, nesse caso o NCD, melhor se explicam os resultados obtidos. Assim, o uso de duas ou mais variáveis explicativas pode contribuir significativamente para o incremento da estimativa do impacto das infestantes no rendimento das culturas. O uso combinado da densidade de infestação e época de convivência das infestantes com a cultura, por exemplo, tende a aumentar a qualidade da estimativa do NCD (PORTUGAL, 2010). Nesse caso, não existe densidade a partir da qual a cultura deve estar isenta das infestantes, mas há densidades que são aceitas até determinado período de convivência.

Uma proposta contemporânea que favorece a aplicação do NDE na Herbologia consiste da utilização de programas de computador (softwares) para mediar as decisões de manejo das plantas daninhas. Esses softwares foram desenvolvidos para avaliar as estratégias de controle de acordo com os seus benefícios agronômicos e econômicos, sendo utilizados com sucesso em ações de manejo integrado em outros países. Cabe destacar que, na utilização desses programas computacionais, parte-se da premissa de que inevitavelmente haverá a decisão do manejo, o qual ocorrerá com base no retorno econômico oportunizado pela medida de controle.

Diversos modelos de softwares foram desenvolvidos e estão disponíveis para os produtores e extensionistas (SKORA NETO, 2010). Destaca-se que, no Brasil, há uma versão de software desenvolvida para o manejo de plantas daninhas denominado 'Sistema de Apoio à Gestão de Infestantes'. Esse programa foi inicialmente criado para mediar as decisões de controle de plantas daninhas na cultura 
do feijão com finalidade didática e de pesquisa (KALSING e VIDAL, 2010). Futuramente, vislumbra-se que as novas versões do programa contemplem diversas espécies de plantas daninhas e informações para o seu manejo integrado.

\section{CONSIDERAÇÕES FINAIS}

Nesta revisão buscou-se avaliar a aplicabilidade do conceito do NDE no manejo de plantas daninhas nas áreas agrícolas. Constatou-se que diversos aspectos têm limitado a utilização desse critério econômico como suporte à decisão do controle dessas plantas na maioria dos sistemas de cultivo. Essas limitações tornam a aplicação da abordagem do NDE na Herbologia uma incógnita, o que acarreta a ausência de um critério para a decisão do manejo das infestantes.

O cenário atual sugere uma mudança de paradigma com relação aos critérios de auxílio ao manejo de plantas daninhas. As ações de manejo integrado dessas espécies apresentam grande importância para diversas culturas e não podem ser comprometidas pela reduzida utilização do NDE. Desse modo, os cientistas da área devem criar alternativas sustentáveis para apoiar tecnicamente a tomada da decisão do controle das plantas daninhas nos agroecossistemas.

Algumas propostas têm buscado aumentar a aplicabilidade do NDE para o manejo de plantas daninhas, principalmente através de métodos que buscam melhorar a estimativa do dano dessas plantas às culturas. Contudo, estratégias alternativas aos níveis de dano, tais como os programas computacionais (softwares), devem ser desenvolvidas para mediar as decisões de manejo das plantas daninhas e favorecer a aplicação do conceito do NDE na Herbologia.

\section{ABSTRACT \\ ECONOMIC THRESHOLD APPLIED TO WEED SCIENCE - REVIEW}

This review addressed the economic weed threshold (EWT) and its application to weed management in crops systems. It discussed the reasons that led to the development of this economic relationship, its function as a criterion for decision to control weeds in integrated management systems, the theory of its scope and magnitude of their values for the weed control, as well as the limitations and prospects of its application in weed science. The development of post-emergence herbicides allowed the adaptation to the EWT concept for weed management. Thus, this threshold was one of the theoretical fundamentals of the integrated weed management, by helping farmers to decide the need for weed control in agricultural areas. In practice, the EWT has not been used in weed management for five reasons: (i) interference relationships were developed for consumer and producer organisms, (ii) naturally occurring polyspecific weeds populations in the most agricultural systems; (iii) lack of stability of biological and economic variables used in the EWT estimation; (iv) the irrelevance of the parameter obtained for crops with low competitive ability; and, (v) the ecological consequences in medium and long term arising from its use to the agroecosystem. Alternative strategies to use the weed thresholds, such as computer softwares, must be developed to aid the decision for weed management.

KEY-WORDS: INTEGRATED WEED MANAGEMENT; THRESHOLD LEVEL; MATHEMATICAL MODELS.

\section{REFERÊNCIAS}

1 AGOSTINETTO, D. et al. Dano econômico como critério na decisão sobre manejo de genótipos de arroz concorrentes em arroz irrigado. Pesquisa Agropecuária Brasileira, v. 40, p. 1-9, 2005.

2 AGUYOH, J.N.; MASIUNAS, J.B. Interference of large crabgrass (Digitaria sanguinalis) with snap beans. Weed Science, v. 51, p. 171-176, 2003.

3 BAUER, T.A.; MORTENSEN, D.A. A comparison of economic and economic optimum thresholds for 2 annual weeds in soybean. Weed Technology, v. 06, p. 228-235, 1992. 
4 BAUER, T. A. et al. Environmental variability associated with economic thresholds for soybeans. Weed Science, v. 39, p. 564-569, 1991.

5 BAYSINGER, J.A.; SIMS, B.D. Giant ragweed (Ambrosia trifida) interference in soybeans (Glicine max). Weed Science, v. 39, p. 358-362, 1991.

6 BAZIRAMAKENGA, R.; LEROUX, G.D. Economic and interference threshold densities of quackgrass (Elytrigia repens) in potato (Solanum tuberosum). Weed Science, v. 46, p. 176-180, 1998.

7 BELL, C.E. Broccoli (Brassica oleracea var botrytis) yield loss from italian reygrass (Lolium perenne) interference. Weed Science, v. 43, p. 117-120, 1995.

8 BOSNIC, A.C.; SWANTON, C.J. Influence of barnyardgrass (Echinochloa crus-galli) time of emergence and density on corn (Zea mays). Weed Science, v. 45, p. 276-282, 1997.

9 BUCKELEW, J.K.; MONKS, D.W. Effect of eastern black nightshade (Solanum ptycanthum) on transplanted plasticulture tomato grade and yield. Weed Science, v. 54, p. 504-508, 2006.

10 BUHLER, D.D. Challenges and opportunities for integrated weed management. Weed Science, v. 50, p. 273-280, 2002.

11 CARDINA, J. et al. Velvetleaf (Abution theophrasti) competition and economic thresholds in conventional and notillage corn (Zea mays). Weed Science, v. 43, p. 81-87, 1995.

12 CARRANZA, P. et al. Competition between ridolfia-segetum and sunflower. Weed Research, v.35, p. 369-375, 1995.

13 CATHCART, R.J.; SWANTON, C.J. Nitrogen management will influence threshold values of green foxtail (Setaria viridis) in corn. Weed Science, v. 51, p. 975-986, 2003.

14 CLEWIS, S.B. et al. Glufosinate-resistant corn interference in glufosinate-resistant cotton. Weed Technology, v. 22, p. 211-216, 2008.

15 COUSENS, R. Theory and reality of weed control thresholds. Plant Protection Quartely, v. 2, p. 19-20, 1987.

16 COUSENS, R. An empirical model relating crop yield to weed and crop density and a statistical comparison with other models. Journal of Agricultural Sciences, v. 105, p. 513-521, 1985.

17 COWBROUGH, M.J. et al. Impact of common ragweed (Ambrosia artemisiifolia) aggregation on economic thresholds in soybean. Weed Science, v. 51, p. 947-954, 2003.

18 FLECK, N.G. et al. Densidade e características morfológicas de plantas de picão-preto na previsão de perdas de rendimento de grãos de soja por interferência. Planta Daninha, v. 20, p. 169-179, 2002a.

19 FLECK, N.G. et al. Dano econômico como critério para tomada de decisão no controle de guanxuma em soja. Planta Daninha, v. 20, p. 421-429, 2002b.

20 FISCHER, D.W. et al. Common lambsquarters (Chenopodium album) interference with corn across the northcentral United States. Weed Science, v. 52, p. 1034-1038, 2004.

21 GALON, L. et al. Níveis de dano econômico para decisão de controle de capim-arroz (Echinochloa spp.) em arroz irrigado (Oryza sativa). Planta Daninha, v. 25, p. 709, 718-2007.

22 GARCIATORRES, L. et al. Modelling the economics of controlling nodding broomrape (Orobanche cernua) in sunflower (Helianthus annuus). Weed Science, v. 44, p. 591-595, 1996.

23 GIBSON, K.D. et al. California arrowhead is a weak competitor in water-seeded rice. Weed Science, v. 49, p. $381-$ $384,2001$.

24 GLIESSMAN, S.R. Agroecologia: processos ecológicos em agricultura. Porto Alegre: Universidade Federal do Rio Grande do Sul, 2001.

25 HAMILL, A.S. Contributions of weed science to weed control and management. Weed Technology, v. 18, p. 15631565, 2004.

26 HAMMOND, C.L. et al. Adoption of integrated pest management tactics by Wisconsin farmers. Weed Technology, v. 20 , p. $756-767,2006$

27 ISI. Institute for Scientific Information. ISI web of knowledge. Disponível em: http://apps.isiknowledge.com. Acesso em: 10.04.2010.

28 KALSING, A. et al. SAGI 1.0: sistema de apoio à gestão de Brachiaria plantaginea na cultura do feijão comum. In: CONGRESSO BRASILEIRO DA CIÊNCIA DAS PLANTAS DANINHAS, 27., Ribeirão Preto, 2010. Resumo.. Londrina: Sociedade Brasileira da Ciência das Plantas Daninhas, 2010. p. 26-29. 
29 KNEZEVIC, S.Z. Interference of redroot pigweed (Amaranthus retroflexus) in corn (Zea mays). Weed Science, v. 42, p. 568-573, 1994.

30 LINDQUIST, J.L. et al. Stability of corn (Zea mays)-foxtail (Setaria spp.) interference relationships. Weed Science, v. $47,195-2000,1999$

31 LINDQUIST, J.L.; KROPFF, M.J. Applications of an ecophysiological model for irrigated rice (Oryza sativa) - Echinochloa competition. Weed Science, v. 44, p. 52-56, 1996.

32 LUTMAN, P.J.W. et al. Prediction of competition between oilseed rape and Stellaria media. Weed Research, v. 40, p. 255-269, 2000.

33 LUTMAN, P.J.W. et al. Investigations into alternative methods to predict the competitive effects of weeds on crop yields. Weed Science, v. 44, p. 290-297, 1996.

34 LUTMAN, P.J.W. et al. The response of 4 spring-sown combinable arable crops to weed competition. Weed Research, v. 34, p. 137-146, 1994.

35 MCDONALD, A.J.; RIHA, S.J. Model of crop: weed competition applied to maize: Abutilon theophrasti interactions. II. Assessing the impact of climate: implications for economic thresholds. Weed Research, v.39, p. 371-381, 1999.

36 NORRIS, R.F. et al. Spatial arrangement, density, and competition between barnyardgrass and tomato: I. Crop growth and yield. Weed Science, v. 49, n. 01, p. 61-68, 2001.

37 NORRIS, R.F. Ecological implications of using thresholds for weed management. Journal of Crop Production, v. 2, p 31-38, 1999.

38 NORRIS, R.F. Case-history for weed competition population ecology - barnyardgrass (Echinochloa-crus-galli) in sugar-beets (Beta vulgaris). Weed Technology, v. 06, p. 220-227, 1992.

39 O'DONOVAN, J.T.; BLACKSHAW, R.E. Effect of volunteer barley (Hordeum vulgare L) interference on field pea (Pisum sativum L) yield and profitability. Weed Science, v. 45, p. 249-255, 1997.

40 ONOFRI, A.; TEI, F. Competitive ability and threshold levels of 3 broadleaf weed species in sinflower. Weed Research, v. 34, p. 471-479, 1994.

41 PESTER, T.A. et al. Secale cereale interference and economic thresholds in winter Triticum aestivum. Weed Science, v. 48, p. $720-727,2000$.

42 PORTUGAL, J. Nível crítico de dano (NCD) de infestantes na cultura do tomate de industria. In: VIDAL, R. A. et al. Nível crítico de infestantes em culturas anuais. Porto Alegre: Evangraf, 2010. p. 57-72.

43 PORTUGAL, J.M.; VIDAL, R.A. Níveis econômicos de prejuízos de plantas infestantes nas culturas agrícolas: conceitos, definições e formas de cálculo. Planta daninha, v. 27, p. 869-877, 2009.

44 RIZZARDI, M.A. et al. Nível de dano econômico como critério para controle de picão-preto em soja. Planta Daninha, v. 21, p. $273-282,2003$.

45 SANYAL, D. et al. Revisiting the perspective and progress of integrated weed management. Weed Science, v. 56, p. 161-167, 2008

46 SKORA NETO, F. Utilização de programas de computador para mediar a gestão de infestantes. In: VIDAL, R.A. et al. Nível crítico de infestantes em culturas anuais. Porto Alegre: Evangraf, 2010. p. 80-89.

47 SATTIN, M. et al. Case-history for weed competition population ecology - velvetleaf (Abutilon theophrasti) in corn (Zea mays). Weed Technology, v. 6, p. 213-219, 1992

48 SMITH, R.F.; ALLEN, W.W. Insect control and the balance of nature. Science, v. 190, p. 38-92, 1954.

49 STERN, V.M. et al. The integrated control concept. Hilgardia, v. 29, p. 81-101, 1959.

50 SWANTON, C.J. et al. Weed thresholds: theory and applicability. Journal of Crop Production, v. 2, p. 9-29, 1999.

51 SWANTON, C.J.; MURPHY, S.D. Weed science beyond the weeds: the role of integrated weed management (IWM) in agroecosystem health. Weed Science, v. 44, p. 437-445, 1996.

52 SWANTON, C.J.; WEISE, S. F. Integrated weed management - the rationale and approach. Weed Technology, v. 5, p. 657-663, 1991.

53 SWINTON, S.M. et al. Estimation of crop yield loss due to interference by multiple weed species. Weed Science, v. 42, p. 103-109, 1994. 
54 THOMAS, W.E. Glyphosate-resistant corn interference in glyphosate-resistant cotton. Weed Technology, v.21, p. 372-377, 2007.

55 TIMMONS, F.L. A history of weed control in the United States and Canadá. Weed Science, v. 53, p. 748-761, 2005.

56 VIDAL, R.A. et al. Interferência e nível de dano econômico de Brachiaria plantaginea e Ipomoea nil na cultura do feijão comum. Ciência Rural, v. 40, p. 1675-1681, 2010b.

57 VIDAL, R.A. et al. Nível crítico de dano de infestantes em culturas anuais. Porto Alegre: Evangraf, $2010 a .133$ p.

58 VIDAL, R.A. Interação negativa entre plantas: inicialismo, alelopatia e competição. Porto Alegre: EVANGRAF, 2010. $130 \mathrm{p}$

59 VIDAL, R.A. et al. Nível de dano econômico de Brachiaria plantaginea na cultura de milho irrigado. Planta Daninha, v.22, p.63-69, 2004.

60 WERNER, E.L. et al. Velvetleaf (Abutilon theophrasti) interference and seed production in corn silage and grain. Weed Technology, v.18, p. 779-783, 2004.

61 WILLIAMS, M.M.; BOYDSTON, R.A. Volunteer potato interference in carrot. Weed Science, v. 54, p. 94-99, 2006.

62 WILLIAMS, M.M. et al. Effect of volunteer potato density on bulb onion yield and quality. Weed Science, v. 52, p. 754758, 2004.

63 WILSON, R.S. et al. Targeting the farmer decision making process: a pathway to increased adoption of integrated weed management. Crop Protection, v. 28, p. 756-764, 2009.

64 ZANIN, G. et al. Estimation of economic thresholds for weed-control in winter-wheat. Weed Research, v. 33, p. 459467, 1993. 